\title{
Electrochemical sensor based on molecularly imprinted membranes at Au@CNTs nanocomposite-modified electrode for determination of prednisolone as a doping agent in sport
}

\author{
Wenhong Wang \\ Department of Physical Education, Jinling Institute of Technology, Nanjing, 211169, China \\ *E-mail: wwh@jit.edu.cn
}

Received: 24 October 2021 / Accepted: 29 November 2021 / Published: 5 January 2022

This study was performed on fabrication of electrochemical sensors based on molecularly imprinted membranes at Au@CNTs nanocomposite-modified electrode for determination of prednisolone (PNS) as a sports doping agent. The Au@CNTs composite was deposited on GCE surface, and $\rho$ aminothiophenol and tetrabutylammonium perchlorate were electropolymerized on Au@CNTs/GCE. Studies on the morphology and microstructure of modified electrodes by SEM and XRD showed that $\mathrm{Au}$ nanoparticles were homogeneously deposited on porous network of CNTs in Au@CNTs nanocomposite, and porous structure of MIP/Au@CNTs/GCE confirming the successful electropolymerization MIP and removal of 2-oxindole template from the body of polymer network. The electrochemical analyses using the CV, DPV and amperometry indicated stable, sensitive and selective performance of MIP/Au@CNTs/GCE to determine PNS. The detection limit, sensitivity and linear range of proposed sensor were found of $0.003 \mu \mathrm{M}, 0.86207 \mu \mathrm{A} / \mu \mathrm{M}$ and $1-210 \mu \mathrm{M}$, respectively, and comparison between the sensing properties of sensor in this work with the reported PNS sensors in literatures demonstrated a wide linear range and a lower detection limit of MIP/Au @CNTs/GCE as PNS sensor that was attributed to the synergistic catalytic effect of MIP and Au@CNTs nanocomposite. The validity and accuracy of MIP/Au@CNTs/GCE for determination of PNS were examined in prepared specimens of blood serum 5 young athletes aged 19 to 23 years who used PNS tablet and the results for determination of PNS in each sample through the amperometry and ELISA techniques indicated to good precision (RSD less than 4.15\%) and good agreement between the ELISA and amperometry measurement on MIP/Au@CNTs/GCE. Therefore, MIP/Au@CNTs/GCE as a reliable PNS sensor may be used to monitor PNS level in blood serum of athletes.

Keywords: Molecularly imprinted polymers; CNTs; Au nanoparticles; Prednisolone; doping agent; blood serum; Amperometry

\section{$\underline{\text { FULL TEXT }}$}


(C) 2022 The Authors. Published by ESG (www.electrochemsci.org). This article is an open access article distributed under the terms and conditions of the Creative Commons Attribution license (http://creativecommons.org/licenses/by/4.0/). 